\title{
Application of Functional Nanoparticle Finishes on Cotton Textiles
}

\author{
Vigneshwaran N*, Bharimalla AK and Arputharaj A \\ ICAR-Central Institute for Research on Cotton Technology, India \\ *Corresponding author: Vigneshwaran N, ICAR-Central Institute for Research on Cotton Technology, Matunga, Mumbai 400019, India \\ submission: 侮June 20, 2018; Published: 監July 13, 2018
}

\begin{abstract}
With the recent advancements in the fields of nanoscience and technology, a new area has been developed within the realm of textile finishing nanofinishing. With an ever-increasing demand for improved functionalities in cotton textiles, nanofinishing helps to realize the required functionalities with the limited amount of additives. Nanoparticles, not only impart the functional properties onto the cotton textiles, but also, reduce the amount of materials required for finishing with uniform distribution of the functionalizing agents on the treated surface. This review focuses on the recent advancements of nanofinishing for cotton textiles with special emphasis on the use of nanoparticles like silver, titania, zinc oxide and silica to impart functional properties like antibacterial, UV-protection, water-repellency and flame retardancy.
\end{abstract}

Keywords: Antibacterial; Cotton textiles; Flame-retardant; Nanofinishing; UV-protection; Water-repellent.

\section{Introduction}

Functional finishes are very much essential in cotton textiles to cover their inherent problems like susceptibility to microbial attack, staining, wrinkling to name a few. Nanofinishing or nanocoating the surface of cotton textiles is one approach to the production of highly active surfaces to have UV-blocking, antimicrobial and self-cleaning properties [1]. Engineered nanoparticles are customsynthesized particles in the size range less than $100 \mathrm{~nm}$, by focusing on desired property enhancement. Conventionally, the basic textile materials or their compositions are changed to achieve the desired property. But, with the help of nanotechnology, it could be achieved by engineering the particle size of various finishing agents. Nanomaterials are generally synthesized by two different approaches: Top-down and Bottom-up. Macroparticles are sizereduced to form nanoparticles by applying mechanical, chemical or bio-energy in case of top-down approach; and, ions/atoms are assembled to form nanoparticles in bottom-up approach. Where top-down approach provides very high yield, bottom-up approach provides product of high quality. Based on the type and nature of application, combination of synthesis protocols are followed for large-scale production [2]. The bottom-up approach permits control of the chemical and structure architecture; however, manual assembly of individual components is clearly prohibitive in time and cost. Top-down technologies provide a progressive interface from the real world (meters, millimetres, micron) to control at the nanometer scale [3]. Eco-friendly and biological synthesis are the latest approaches to overcome the eco-toxicity and safety issues. Plants, algae, fungi, bacteria, and viruses have been used for production of low-cost, energy-efficient, and nontoxic metallic nanoparticles [4].

Stabilization of nanoparticles is a major issue while dealing with their applications in diversified fields like sensors, filters, composites and smart textiles. Due to high surface energy, the synthesized nanoparticles tend to aggregate/agglomerate among themselves to reduce their instability. Hence, there is a requirement for addition of stabilizer or protective agents into the nanoparticle system to maintain their size dependent properties. Some of the size dependent properties include surface plasmon resonance, quantum confinement effect, superconductivity, super-paramagnetism, and so on. Moreover, the morphology and size distribution of prepared nanoparticles vary with the concentration of the stabilizer and type of the precursors [5]. In this review, we focus on the use of nanoparticles in textile finishing, their application protocols and their potential for future expansion.

\section{Application protocols}

The incorporation of nanoparticles on the surface of textile materials inherits a major challenge in terms of durability and efficacy. In most cases, nanoparticles on its own do not have good affinity towards the textile materials. Either they need to be surface modified or applied using a binder to impart the affinity $[6,7]$. To overcome these problems, many researchers including our team are working on in-situ formation of nanoparticles on the surface 
of textile materials by utilizing their macro and mesopores as templates. Figure 1 shows various alternatives for application of nanoparticles on the surface of textile materials. For in-situ processing: in case of cellulosic and woolen textile materials, the basic substrate is swollen by chemical treatment and the openedup pores are used as template for synthesis of nanoparticles. Once the chemical environment is withdrawn, the opened-up pores collapse, holding the nanoparticles inside the system. For synthetic polymers, nanoparticles are added during extrusion, so that, the polymeric filament holds them very tightly. Alternatively, surface etching of the textile materials are done to impart nano-finishing on the surface. For ex-situ processing: the simplest way is by paddry-cure method, wherein, a polymeric binder applied along with the nanoparticles treatment to the textile materials [8]. Also, it is possible to surface modify the nanoparticles and chemically link to the surface of the textile materials. In recent developments, it is reported to impart nanofinishes using electro-spraying wherein, with the help of very high voltage (up to $80 \mathrm{kV}$ ), polymeric solution is converted to nanoparticles/nanofibers and deposited on the surface of textile materials [9].

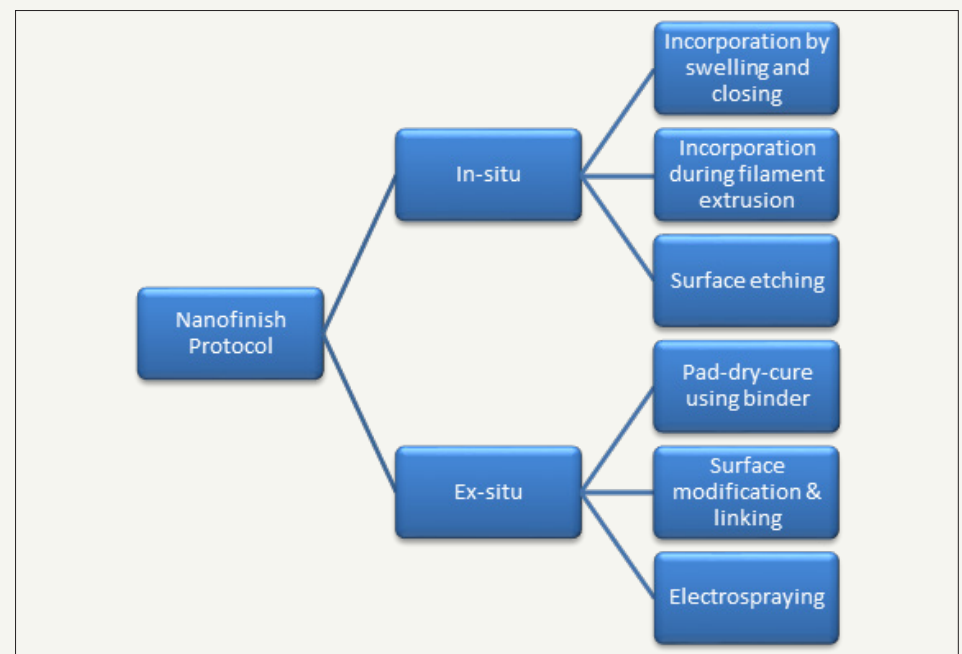

Figure 1: Application protocols for nanoparticles onto textile materials.

\section{Types of finishes applied on cotton textile products}

Nanofinishing refers to the use of nanotechnology on the textile products to impart functional properties. It refers to both the external application of nanomaterials on the surface and in-situ preparation of nanostructures in the textile materials.

Antimicrobial finish: Antimicrobial finished textiles are required not only in medical field, but also for use in hotels, hostels, railways, home furnishings and other public places. In conventional silver treatment or nano-Ag treatment, the treated textiles achieve their antimicrobial activity mainly due to the release of silver ions. Hence, the antimicrobial efficacy of the treated textile is related to the capability of antimicrobial agents to release silver ions [10]. Both Ag ions and nano-Ag can participate in the intermolecular interactions with the cell membrane of bacteria. In some literature, it is reported that the nano-Ag smaller than $10 \mathrm{~nm}$ can penetrate into the microbial cell and bind to the thiol groups of enzymes and nucleic acids [11]. Alternatively, in the presence of oxygen, the nano-Ag may catalytically accelerate the formation of reactive oxygen species (ROS) that are highly toxic to the microbial cells [12]. In some cases, the nanoparticles interrupt the process of DNA replication and causes cell mortality. The various mechanisms of antibacterial activity by nanomaterials are given in Figure 2 .

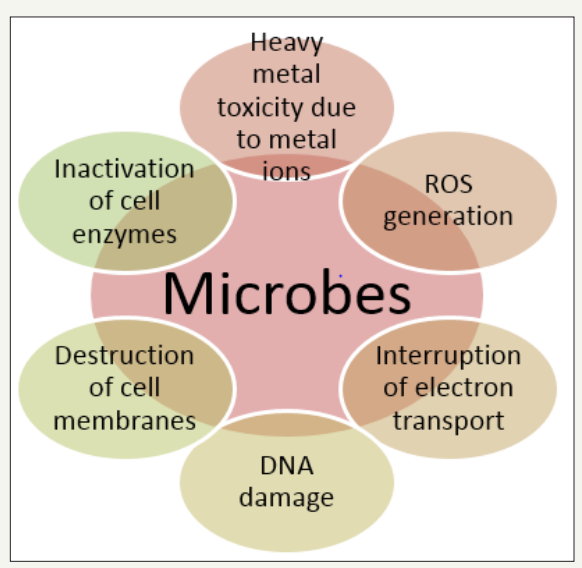

Figure 2: Possible mechanisms of antimicrobial activity by nanomaterials on microbes. 
UV protection finish: Ultraviolet (UV) rays, part of the energy that comes from the sun, can burn our skin and cause cancer. UV rays are made up of three types: ultraviolet $A$ (UVA), ultraviolet $B$ (UVB), and ultraviolet C (UVC). Though UVC is the most dangerous type, it can't penetrate Earth's ozone layer. UVA and UVB do penetrate the ozone layer and reach the surface of Earth. Both UVA and UVB are reported to cause skin cancer, including melanoma. When straight UV light falls onto a textile material, a part of the UV light is reflected, the other part gets absorbed. The absorbed light is transmitted through the cloth as shown in Figure 3(a). Textile materials can provide protection against harmful effects of UV radiation if the necessary attention is paid while engineering the basic design and structure of the material. Metal oxides such as $\mathrm{ZnO}, \mathrm{TiO}_{2}$ in nano form absorb UV light and act as a UV protecting agents for textile material. The band gap energy should be in range of $3-3.5 \mathrm{eV}$ to act as good UV absorption material. The required band gap can be achieved simply reducing the size of a particle as given in Figure 3(b). The band gap increases from $3.19 \mathrm{eV}$ in case of micro-ZnO to $3.32 \mathrm{eV}$ in Nano ZnO. Table 1 shows the band gap energies for different metal oxides [13]. Doping of metal oxides [14] and synthesis of hybrid nanomaterials are the methods to produce tailor-made material for specific end use. Sol-gel method of production of nano-titania [15] and in situ generation of nano$\mathrm{ZnO}$ on cotton fabric [16] result in durable UV protective materials. In sol-gel method, the metal precursor undergoes rapid hydrolysis to produce the metal hydroxide solution, followed by immediate condensation resulting in three-dimensional gels. During drying, it forms the crystalline metal oxide nanoparticles. In case of in situ process, the metal precursors enter the swollen cotton fibres and subjected to double decomposition and hydrolysis resulting in nanoparticles formation inside the pores.

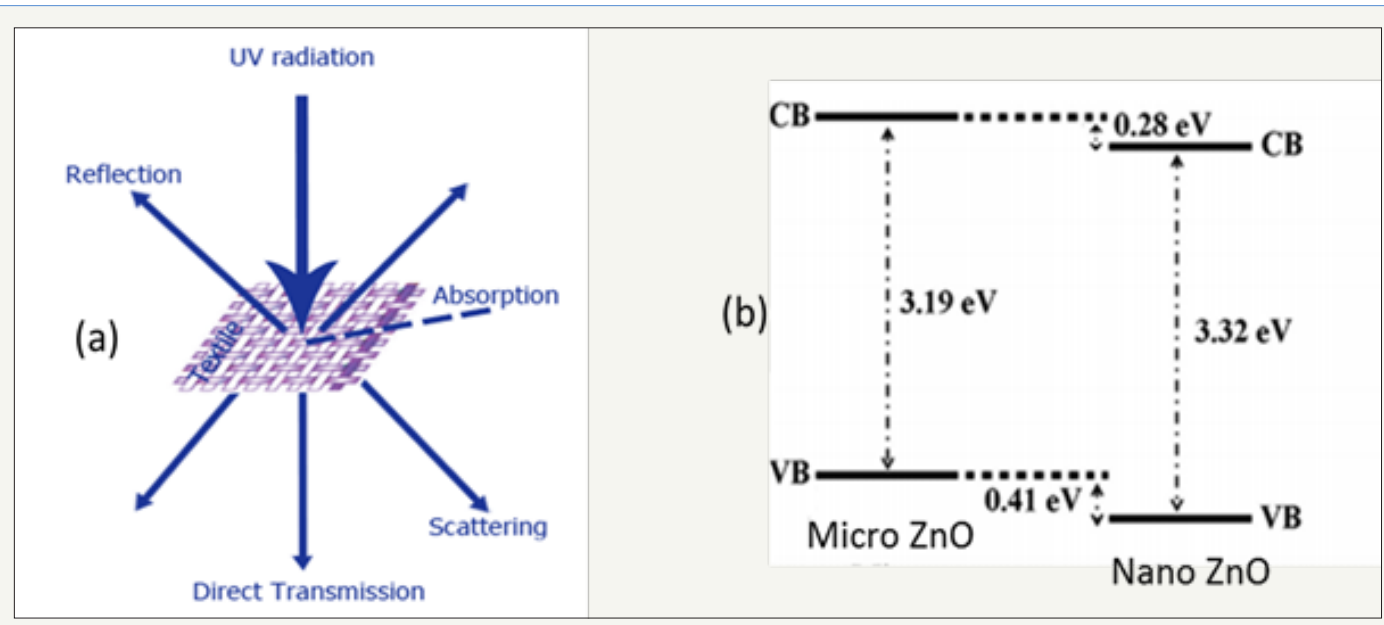

Figure 3: Textile fabric interaction with light (a) and influence of particle size in band gap energy of $\mathrm{ZnO}$ (b) [14]. VB indicates valence band and $\mathrm{CB}$, conduction band.

Table 1: Band gap energy of different metal oxides [13].

\begin{tabular}{|c|c|}
\hline Nature of Metal Oxide & Band Gap Energy (Ev) \\
\hline $\mathrm{ZnO}$ & 3.2 \\
\hline $\mathrm{TiO}_{2}$ (Anatese) & 3.2 \\
\hline $\mathrm{TiO}_{2}$ (Rutile) & 3.0 \\
\hline $\mathrm{ZrO}_{2}$ & 5.0 \\
\hline $\mathrm{Fe}_{2} \mathrm{O}_{3}$ & 2.3 \\
\hline $\mathrm{Cu}_{2} \mathrm{O}$ & 2.2 \\
\hline $\mathrm{SnO}_{2}$ & 3.8 \\
\hline
\end{tabular}

Water repellence finish: Cotton, the king of fibres, is the best suitable substrate for use in fabrics for necessary comfort. But, the hydrophilicity of the cotton fibres makes them non-usable where there is requirement for water repellency. In this regards, researchers are trying to mimic the hydrophobic surface structure of the lotus leaf (LOTUS EFFECT ${ }^{\circledR}$ ) by modifying the surface of cotton in the nano-scale. Water-repellent finish is used to protect textile fabrics from wetting, without adversely affecting the other properties such as air permeability of the finished fabrics. This is achieved by reducing the free energy at the textile surface. The degree to which the amount of water can be attached to a surface is determined by the surface free energy. Surface with lower surface energy could not be wetted with water [17]. Surface tension of textile surface should be lower than that of the liquid to achieve active repellent surface. Surfaces that exhibit low interactions with liquids are referred to as low energy surfaces, i.e., if the bonding forces between the fabric surface and the liquid are less than the internal cohesive interactions within the liquid, the drop will not spread. Low energy textile surface can be produced via chemical reaction of the repellent material with the fabric surface. Examples of these are fatty acids, waxes and fluorocarbon treated surfaces.

Super hydrophobic surfaces such as leaves of the lotus plant are extremely difficult to wet. The contact angles of a water droplet exceed $150^{\circ}$ and the roll-off angle is lesser than $10^{\circ}$. This is referred to as the Lotus effect [18]. Dirt particles are picked up by water droplets due to a complex micro and nano scopic architecture of the surface, which minimizes adhesion. One of the approaches for developing a super hydrophobic surface consists of a two-step 
process: producing nanostructure surface followed by a treatment with a fluorocarbon containing polymer or silane compounds [19]. Unconventional new approaches for the design of super hydrophobic surfaces are focused on the surface functionalization of silica nanoparticles with non-fluorinated alkylsilanes [20] or fluorosilanes [21]. Cotton fabrics treated with silica nanoparticels (synthesized via sol-gel process) in combination with very low amount of water-repellent agent exhibits super-hydrophobicity with a contact angle above 130degree [22].

Flame retardant finish: During any fire accident, the fibres that caught fire after initial ignition were cotton followed by polyamide, filament acetate, viscose and others. Thus, the cellulosic garments were reported to be the most flammable [23]. Cellulose undergoes thermal decomposition at temperatures above $300^{\circ} \mathrm{C}$. It forms not only water and carbon dioxide, but also carbon monoxide, methanol, ethanol, acetone, formic acid, acetic acid, and other products which will ignite and burn with an adequate supply of air. It is generally assumed that an effective flame-retardant finish controls pyrolysis by inducing the cellulose to carbonize through dehydration at temperatures below $300^{\circ} \mathrm{C}$ [23]. The limiting oxygen index (LOI) technique provides a quantitative measure of reduced flammability for fabrics over broad ranges of chemical treatment [24]. Recent research is focused on use of nanoparticles to impart flame-retardant properties. Nano zinc oxide applied on bleached jute fabric to imparted fire retardant property. Nano zinc oxide was prepared from aqueous Zinc acetate by co-precipitation method in the presence of $\mathrm{NaOH}$ followed by calcination at $600{ }^{\circ} \mathrm{C}$. It was then applied on the jute fabric using an aqueous solution of Potassium Methyl Siliconate as dispersing media by pad-dry method. Application of $0.01 \%$ of nano $\mathrm{ZnO}$ on jute fabric showed reasonably good fire retardant performance [25]. Also, it was reported to impart multifunctional properties by using hybrid nanoparticles. Cotton fabrics with good water-oil-repellence and flame-retardant properties with relatively durable properties could be produced using nanosols containing guanidine dihydrogen phosphate and urea, together with tetraethylorthosilicate and hexadecyltrimethoxysilane as precursors, and lower concentrations of Guard $\mathrm{AFB}^{\circledR}$ as commercial water-oil-repellent agent [26].

\section{Safety and ecotoxicology}

The washing of nano-Ag treated textiles potentially releases both dissolved and particulate silver, and, few of the Ag released are of the particles having size larger than 450nm [27]. Hence, life cycle assessment is very much essential to know the movement of nanomaterials and their status/fate once out of control into the environment. A cradle-to-grave life cycle assessment [28] was reported to compare nanosilver T-shirts with conventional T-shirts with and without biocidal (triclosan) treatment. The "cradle-togate" climate footprint of the production of a nanosilver T-shirt was $2.70 \mathrm{~kg}$ of $\mathrm{CO}_{2}$-equiv for the flame spray pyrolysis method. Production of conventional T-shirts with and without the biocide triclosan has emissions of $2.55 \mathrm{~kg}$ of $\mathrm{CO}_{2}$-equiv (contribution from triclosan insignificant). Consumer behavior considerably affects the environmental impacts during the use phase. Lower washing frequencies compensated for the increased climate footprint of nanosilver T-shirt production. The toxic releases from washing and disposal in the life cycle of T-shirts appear to be of minor relevance [28].

The safety issues need to be addressed in laboratories, industries and also in their site of use. The awareness need to be created among the customers for their safe handling and disposal. Also, a crucial point of future developments in nanotechnology is the consideration of the ethical, legal, and societal implications of this technology. The means of introducing nanotechnology based products into the society, and their relevant policies are to be discussed with various stakeholders. The means of addressing ethical, legal, and social issues will determine the trust by the public and the future of nanotechnology based innovation.

\section{International standards for nanotechnology}

International Standardization Organization (ISO)/Technical Committee (TC) 229 is working on the development of standards for Nanotechnologies [29]. Standardization in the field of nanotechnologies that includes either or both of the following:

A. Understanding and control of matter and processes at the nanoscale, typically, but not exclusively, below 100 nanometres in one or more dimensions where the onset of size-dependent phenomena usually enables novel applications,

B. Utilizing the properties of nanoscale materials that differ from the properties of individual atoms, molecules, and bulk matter, to create improved materials, devices, and systems that exploit these new properties.

Specific tasks include developing standards for: terminology and nomenclature; metrology and instrumentation, including specifications for reference materials; test methodologies; modelling and simulations; and science-based health, safety, and environmental practices.

Other leading standards setting organizations and their relevant nanotechnology committees are:

A. ASTM (formerly known as the American Society for Testing and Materials) International's Committee E56 (Nanotechnology) [30].

B. International Electrotechnical Commission Technical Committee 113 (Nanotechnology Standardization for Electrical and Electronics Products and Systems) [31].

\section{Conclusion}

Nanotechnology is revolutionizing the way in which the functional properties are imparted to the textile materials. Cotton, the king of natural fibres, requires nanotechnological interventions to achieve the functionalities like antibacterial, UV protecting, super hydrophobic and flame retardancy. Nanofinishing also helps to impart these properties without affecting the inherent comfort nature of cotton. Also, the amount of material required to impart the functional property is relatively less due to its nano-size range. But, 
caution needs to be exercised to avoid excess and improper usage of the nanomaterials and to restrict the entry of waste nanoparticles into the environment.

\section{References}

1. Yadav A, Prasad V, Kathe AA, Raj S, Yadav D, et al. (2006) Functional finishing in cotton fabrics using zinc oxide nanoparticles. Bull Mater Sci 29(6): 641-645.

2. Thakkar KN, Mhatre SS, Parikh RY (2010) Biological synthesis of metallic nanoparticles. Nanomedicine: Nanotechnology, Biology and Medicine 6(2): 257-262.

3. Mazzola L (2003) Commercializing nanotechnology. Nat Biotechnology 21(10): 1137-1143.

4. Tsuzuki T (2009) Commercial scale production of inorganic nanoparticles. Int J Nanotechnol 6(5/6): 567-578.

5. Huang H, Yang X (2004) Synthesis of polysaccharide-stablized gold and silver nanoparticles: a green method. Carbohydr Res 339(15): $2627-$ 2631.

6. Kim HW, Kim BR, Rhee YH (2010) Imparting durable antimicrobial properties to cotton fabrics using alginate-quaternary ammomium complex nanoparticles. Carbohydr Polym 79(4): 1057-1062.

7. Montazer M, Alimohammadi F, Shamei A, Rahimi MK (2012) Durable antibacterial and cross-linking cotton with colloidal silver nanoparticles and butane tetracarboxylic acid without yellowing. Colloids and Surfaces B: Biointerfaces 89: 196-202.

8. Vigneshwaran N, Kumar S, Kathe AA, Varadarajan PV, Prasad V (2006) Functional finishing of cotton fabrics using zinc oxide-soluble starch nanocomposites. Nanotechnology 17(20): 5087-5095.

9. Prabu GTV, Chattopadhyay SK, Patil PG, Arputharaj A, Mandhyan PK, et al. (2017) Moisture management finish on cotton fabric by electrospraying. Textile Research Journal 87(17): 2154-2165.

10. Nowack B, Krug HF, Height M (2011) 120 Years of nanosilver history: Implications for policy makers. Environ Sci Technol 45(17): 1177-1183.

11. Feng QL, Wu J, Chen GQ, Cui FZ, Kim TN, et al. (2000) A mechanistic study of the antibacterial effect of silver ions on Escherichia coli and Staphylococcus aureus. J Biomed Mater Res 52(4):662-668.

12. Marambio-Jones C, Hoek EMV (2010) A review of the antibacterial effects of silver nanomaterials and potential implications for human health and the environment. J Nanopart Res 12(5): 1531-1551.

13. Ola O, Maroto-Valer MM (2015) Review of material design and reactor engineering on $\mathrm{TiO}_{2}$ photocatalysis for $\mathrm{CO}_{2}$ reduction. J Photochem Photobiol C: Photochem Rev 24:16-42.

14. Kamarulzaman N, Kasim MF, Rusdi R (2015) Band gap narrowing and widening of $\mathrm{ZnO}$ nanostructures and doped materials. Nanoscale Res Lett 10(1): 1034

15. Hafizah N, Sopyan I (2009) Nanosized $\mathrm{TiO}_{2}$ photocatalyst powder via sol-gel method: Effect of hydrolysis degree on powder properties. Int J Photoenergy 2009(8): 1-8.

16. Arputharaj A, Vigneshwaran N, Shukla SR (2017) A simple and efficient protocol to develop durable multifunctional property to cellulosic materials using in situ generated nano-ZnO. Cellulose 24(8): 3399-3410.

17. Wang H, Shi H, Li Y, Wang Y (2014) The effects of leaf roughness, surface free energy and work of adhesion on leaf water drop adhesion. PLOS ONE 9(9): 1-10.

18. Latthe SS, Tereshima C, Nakata K, Fujishima A (2014) Super hydrophobic surfaces developed by mimicking hierarchical surface morphology of lotus leaf. Molecules 19(4): 4256-4283.

19. Bravo L, Zhai Z, Wu RE, Cohen MF, Rubner, (2007) Transparent super hydrophobic films based on silica nanoparticles. Langmuir 23(13): 7293-7298.

20. Gao Q, Zhu Q, Guo Y, Yang CQ (2009) Formation of highly hydrophobic surfaces on cotton and polyester fabrics using silica sol nanoparticles and nonfluorinated alkylsilane. Ind Eng Chem Res 48(22): 9797-9803.

21. Przybylak M, Maciejewski H, Dutkiewicz A, Dąbek I, Nowicki M (2016) Fabrication of super hydrophobic cotton fabrics by a simple chemical modification. Cellulose 23(3): 2185-2197.

22. Bae GY, Min BG, Jeong YG, Lee SC, Jang JH (2009) Superhydrophobicity of cotton fabrics treated with silica nanoparticles and water-repellent agent. J Colloid Inter Sci 337(1): 170-175.

23. Aenishanslin R, Guth C, Hofmann P, Maeder A, Nachbur H (1969) A new chemical approach to durable flame-retardant cotton fabrics. Text Res J 39(4): 375-381.

24. Willard JJ, Wondra RE (1970) Quantitative evaluation of flame-retardent cotton finshes by the limiting oxygen index (LOI) technique. Text Res J 40(3): 203-210.

25. Samanta AK, Bhattacharyya R, Jost S, Basu S, Basu G, (2017) Fire retardant finish of jute fabric with nano zinc oxide. Cellulose 24(2): 1143-1157.

26. Onar N, Mete G (2016) Development of water-, oil-repellent and flameretardant cotton fabrics by organic-inorganic hybrid materials. J Text Inst 107(11): 1164-1177

27. Benn T, Cavanagh B, Hristovski K, Posner JD, Westerhoff P (2010) The release of nanosilver from consumer products used in the home. J Environ Quality 39: 1875-1882.

28. Walser T, Demou E, Lang DJ, Hellweg S (2011) Prospective environmental life cycle assessment of nanosilver t-shirts. Environmental Science and Technology 45(10): 4570-4578.

29. https://www.iso.org/committee/381983/x/catalogue/

30. https://www.astm.org/Standards/nanotechnology-standards.html

31.http://www.iec.ch/dyn/www/f?p=103:29:0::::FSP_ORG_ID,FSP_LANG_ ID: 1315,25

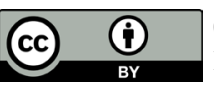

Creative Commons Attribution 4.0

International License

For possible submissions Click Here

\section{Submit Article}

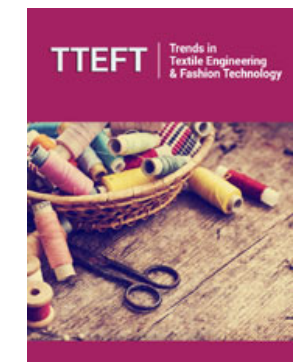

Trends in Textile Engineering \& Fashion Technology

\section{Benefits of Publishing with us}

- High-level peer review and editorial services

- Freely accessible online immediately upon publication

- Authors retain the copyright to their work

- Licensing it under a Creative Commons license

- Visibility through different online platforms 